\title{
Use of Interpreters by Physicians
}

\author{
Lenny López, MD, MPH \\ ${ }^{1}$ Mongan Institute for Health Policy, and Disparities Solutions Center, Massachusetts General Hospital, Boston, MA, USA; ${ }^{2}$ Department of General \\ Medicine, Massachusetts General Hospital, Boston, MA, USA; ${ }^{3}$ Department of Medicine, Brigham and Women's Hospital, Boston, MA, USA.
}

J Gen Intern Med 30(11): 1590

DOI: $10.1007 / \mathrm{s} 11606-015-3474-4$

(c) Society of General Internal Medicine 2015

I thank Dr. Jacobs and colleagues for their interest in my published study. Indeed, the field of study regarding limited English proficiency (LEP) is of vital importance for ensuring high quality care as the US continues to become more heterogeneous from continued immigration from many parts of the world. As noted by the respondents, the published study is a retrospective analysis with concomitant limitations noted in the discussion section of the paper. I agree that the optimal method of identifying LEP patients should be a two-part process to ensure high accuracy. Our institution has a similar process. In the methods section, it is stated: "Self-reported preferred language was collected at registration by trained registrar personnel with standardized scripts. In addition, the nurse intake form on the hospital inpatient units collected and/or confirmed language preference at the time of admission." The possibility of misclassification is also discussed, but I believe the rate to be low for the reasons listed in the text of the paper.
In the discussion section of the paper, I addressed the heterogeneity that underlies LEP status. The LEP status continuum may be affected by other important factors, such as patients' length of residence in the US and preferences for interpreter use. I agree with Dr. Jacobs et al. that future studies will need to account for this complexity, allowing for more precise and accurate measurement of LEP status and interpreter need. This includes language concordance between provider and patient as well as the use of family members with high levels of health literacy in both languages, who might be involved in clinician and patient communication. I would like to emphasize that inpatient care is a continuum of clinical care encounters with both clinical and non-clinical staff. As a result, it is not enough to have interpreter use only at the time of admission or possibly only at discharge. Future efforts to improve inpatient LEP quality and patient safety need to prioritize a goal of providing high quality interpreter service, most likely remotely, for all of the multiple inpatient clinical encounters that occur during a hospitalization. This is the standard we owe all of our patients to ensure that we eliminate language as a barrier to the highest quality healthcare.

Lenny López, MD

Published online July 30, 2015 